\title{
A importância da Atenção Primária à Saúde na detecção e terapêutica de sintomas depressivos em idosos
}

The importance of Primary Health Care in the detection and treatment of depressive symptoms in the elderly

La importancia de la Atención Primaria de Salud en la detección y tratamiento de los síntomas depresivos en los ancianos

Recebido: 30/01/2021 | Revisado: 04/02/2021 | Aceito: 06/02/2021 | Publicado: 14/02/2021

\author{
Mirlane Pereira Soares \\ ORCID: https://orcid.org/0000-0002-0468-6295 \\ Universidade Federal do Maranhão, Brasil \\ E-mail: mirlane.soares@ outlook.com \\ Sueli de Souza Costa \\ ORCID: https://orcid.org/0000-0003-4127-7324 \\ Universidade Federal do Maranhão, Brasil \\ E-mail: scsueli@gmail.com \\ Izolda Souza Costa \\ ORCID: https://orcid.org/0000-0003-0899-6845 \\ Universidade Federal do Maranhão, Brasil \\ E-mail: izolda.costa@hotmail.com \\ Nilson de Jesus Pereira Batalha Júnior \\ ORCID: https://orcid.org/0000-0002-6325-5655 \\ Universidade Federal do Maranhão, Brasil \\ E-mail: nilsonbatalha@gmail.com
}

\begin{abstract}
Resumo
O aumento expressivo do número de idosos é um acontecimento global, que vêm ocorrendo de maneira constante. Dados remontam que essa população se aproximará de 2 bilhões de pessoas até 2050. Associado a esse crescimento, sintomas depressivos e a depressão, propriamente dita, revelam preocupação com uma epidemia incidente, principalmente no grupo etário dos idosos, que costumeiramente negligencia os sintomas com a crença de serem inerentes ao processo natural do envelhecimento. O presente estudo é uma revisão integrativa da literatura e teve como objetivo demonstrar a importância da atenção primária à saúde na detecção de sintomas depressivos em idosos, conhecer os agravos relacionados com os sintomas, identificar o melhor instrumento para detecção precoce da problemática e descrever as terapêuticas empregadas nos idosos com sintomas depressivos. A busca foi realizada nas bases de dados eletrônicas SCIELO, LILACS e BIREME, incluindo trabalhos publicados entre 2011 e 2019, em língua portuguesa. A seleção dos estudos, extração de dados e validação foram realizadas de forma independente por dois autores, obtendo amostra de 37 artigos. Constatou-se, através dos dados analisados, os principais métodos utilizados para rastreio dos sintomas depressivos, comorbidades mais associadas relatados na literatura e os principais métodos abordados para tratamento. Assim, foi possível estruturar de forma sistemática os relatos mais recentes da problemática na atenção primária à saúde e lançar base para elucidação, escolha e possível padronização dos métodos de rastreio.
\end{abstract}

Palavras-chave: Depressão; Idoso; Atenção primária à saúde.

\begin{abstract}
The expressive increase in the number of elderly people is a global event, which has been occurring steadily. Data show that this population will approach 2 billion people by 2050. Associated with this growth, depressive symptoms and depression, properly speaking, reveal concern about an incident epidemic, mainly in the age group of the elderly, who usually neglect symptoms with belief that they are inherent to the natural aging process. The present study is an integrative literature review and aimed to demonstrate the importance of primary health care in detecting depressive symptoms in the elderly, to know the problems related to the symptoms, to identify the best instrument for early detection of the problem and to describe the therapies used in the elderly with depressive symptoms. The search was carried out in the electronic databases SCIELO, LILACS and BIREME, including works published between 2011 and 2019, in Portuguese. The selection of studies, data extraction and validation were carried out independently by two authors, obtaining a sample of 37 articles. It was found, through the data analyzed, the main methods used to screen for depressive symptoms, the most associated comorbidities reported in the literature and the main methods addressed
\end{abstract}


for treatment. Thus, it was possible to systematically structure the most recent reports of the problem in primary health care and lay the ground for elucidation, choice and possible standardization of screening methods.

Keywords: Depression; Old man; Primary health care.

\section{Resumen}

El expresivo aumento en el número de personas mayores es un evento global, que se ha venido produciendo de manera constante. Los datos muestran que esta población se acercará a los 2.000 millones de personas en 2050. Asociados a este crecimiento, los síntomas depresivos y la depresión, propiamente hablando, revelan la preocupación por una epidemia incidente, principalmente en el grupo de edad de los ancianos, que suelen descuidar los síntomas con la creencia de que son inherentes al proceso de envejecimiento natural. El presente estudio es una revisión integradora de la literatura y tuvo como objetivo demostrar la importancia de la atención primaria de salud en la detección de síntomas depresivos en el anciano, conocer los problemas relacionados con los síntomas, identificar el mejor instrumento para la detección precoz del problema y describir la terapias utilizadas en ancianos con síntomas depresivos. La búsqueda se realizó en las bases de datos electrónicas SCIELO, LILACS y BIREME, incluyendo trabajos publicados entre 2011 y 2019, en portugués. La selección de estudios, extracción de datos y validación fueron realizadas de forma independiente por dos autores, obteniendo una muestra de 37 artículos. Se encontró, a través de los datos analizados, los principales métodos utilizados para el cribado de síntomas depresivos, las comorbilidades más asociadas reportadas en la literatura y los principales métodos abordados para el tratamiento. Así, fue posible estructurar sistemáticamente los informes más recientes del problema en la atención primaria de salud y sentar las bases para el esclarecimiento, elección y posible estandarización de los métodos de detección.

Palabras clave: Depresión; Anciano; Primeros auxilios.

\section{Introdução}

O aumento da população idosa é um acontecimento global constante, que vem ocorrendo como reflexo de melhoria na qualidade de vida (Magalhães et al., 2016; Souza; Ferreira, Santos, Oliveira, \& Andrade, 2017; Medeiros, Coura, \& Ferreira, 2017). Dados apontam que em 2025 essa população chegará a um total de aproximadamente 1,2 bilhões de indivíduos e, até 2050, existirá 2 bilhões de pessoas nessa faixa etária, concentrando-se em sua maioria nos países em desenvolvimento (Souza et al., 2017; Uchoa, Chaves, Botelho, Polaro, \& Oliveira, 2019).

Nessa realidade de envelhecimento populacional, dilemas de saúde que atingem os idosos, como as doenças crônicas, ganham mais destaque (Hellwig, Munhoz, \& Tomasi, 2016; Silva et al., 2017; Rêgo et al., 2017). Associado a esse dilema, os sintomas depressivos merecem especial notoriedade, pois cerca de $8 \%$ a $16 \%$ das pessoas nessa faixa etária são acometidas por esse problema (Previato, Labegalini, Carreira, \& Baldissera, 2016; Gullich, Duro, \& Cesar, 2016).

Os sintomas depressivos, costumeiramente, são considerados como uma decorrência natural do processo de envelhecimento, negligenciando seu papel como possível causador de morbidades e diminuição da saúde dos idosos, de seus familiares e cuidadores (Silva et al., 2017; Abrantes et al., 2019). Os sintomas depressivos não se apresentam de forma homogênea, sofrendo variações que dependem de fatores biopsicossociais (Bretanha et al., 2015; Piani et al., 2016; Gouveia et al., 2020); fato que requer ainda mais atenção.

O rastreio da depressão deve ser precoce e focado em sintomas como mudanças de humor, desânimo, isolamento social, condição financeira e familiar precárias, ausência de afeto positivo, irritabilidade, apatia, ansiedade, alterações de sono e do apetite (Ramos, Carneiro, Barbosa, Mendonça, \& Caldeira, 2015; Lino et al., 2016). Para isto, instrumentos simples e de baixo custo para investigação são empregados, como Escala de Depressão Geriátrica (EDG), Avaliação Geriátrica Ampla (AGA), Mini Exame do Estado Mental (MEEM), Escala de Lawton e Brody, Índice de Katz, Escala Brazil Old Age Schedule (BOAS) e o Protocolo de Identificação do Idoso Vulnerável (VES-13), a fim de reconhecer alterações e evitar os prováveis riscos (Lino et al., 2016; Previato et al., 2016; Uchoa et al., 2019; Didoné et al., 2020).

Esse dilema necessita de estudos e aprimoramentos constantes, visto que os profissionais da área da saúde, em especial os da atenção básica, devem prevenir, identificar, diagnosticar, tratar e encaminhar as pessoas idosas que apresentarem qualquer alteração que remontem sintomas depressivos (Magalhães et al., 2016; Soares, Silva, Santos, \& Silva, 2017; Rêgo et al., 2017), pois afetam diretamente a qualidade de vida da pessoa idosa (Silva et al., 2017; Abrantes et al., 2019). 
Desta forma, este estudo visou demonstrar a importância da atenção primária à saúde na detecção de sintomas depressivos em idosos, no período de 2011 a 2019, conhecer os agravos relacionados com os sintomas, identificar o melhor instrumento para detecção precoce da problemática e descrever as terapêuticas empregadas nos idosos com sintomas depressivos.

\section{Metodologia}

O presente trabalho consiste em uma revisão integrativa da literatura (Pereira, Shitsuka, Parreira, \& Shitsuka, 2018). Cinco etapas norteadoras foram adotas na realização da pesquisa, sendo: 1) Elaboração do conceito principal norteador da pesquisa: "Qual a importância da atenção primária à saúde na detecção e terapêutica de sintomas depressivos em idosos, melhor escala para avaliação, sintomas depressivos mais recorrentes e seus agravos relacionados, e terapêutica empregada?; 2) Busca ou amostragem na literatura de trabalhos relacionados ao tema publicados nos últimos nove anos (2011 - 2019), em concordância aos critérios de inclusão e exclusão definidos; 3) Coleta de dados; 4) Análise crítica dos trabalhos relevantes; e 5) Apresentação e discussão dos resultados.

A busca foi realizada nas bases de dados eletrônicas Scientific Eletronic Library Online (SCIELO), Literatura LatinoAmericana do Caribe em Ciências da Saúde (LILACS) e Biblioteca Regional de Medicina (BIREME). As referências que preencheram os critérios de inclusão foram avaliadas com relação ao conteúdo publicado e estrutura do trabalho. A seleção dos descritores utilizados no processo de revisão foi efetuada mediante consulta aos Descritores de Assunto em Ciências da Saúde (DECs). Nas buscas, os seguintes descritores, em língua portuguesa, foram considerados: "depressão" (descritor 1); "idoso" (descritor 2); "atenção básica" (descritor 3).

Foram empregues etapas e descritores em categorias para a seleção dos artigos nas bases de dados, conforme apontado a seguir: 1) SCIELO e LILACS: a) "depressão" e "idoso"; b) "depressão" e "atenção básica"; c) "idoso" e "atenção básica"; d) "depressão" e "idoso" e "atenção básica"; e 2) BIREME: a) "depressão" e "idoso" e "atenção básica", sendo feito rodada diferente em virtude da ausência de resultados com o esquematizado para as bases anteriores.

Foram definidos os critérios de inclusão e exclusão adotados para a seleção dos estudos. Assim, os critérios de inclusão dos artigos foram: tema apropriado ao nível de atenção à saúde da pesquisa; ideias claras, objetivas e condizentes com o estudo; e trabalhos em acordo com a faixa etária proposta. Por sua vez, foram excluídos: publicações que fogem ao tema; trabalhos em língua estrangeira; e artigos em duplicidade na mesma plataforma e/ou diferentes.

Para avaliação dos trabalhos selecionados, elaborou-se um instrumento sistemático para a coleta das informações de forma independente por dois autores, visando responder à questão norteadora do estudo, no qual os dados relevantes encontrados foram sintetizados e posteriormente dispostos em um quadro sinóptico contendo o ano do estudo, autor, título, instrumento de rastreio, terapêutica e o enfoque do estudo. Discrepâncias na escolha dos artigos a serem incluídos foram resolvidas através de discussão entre os autores do presente trabalho.

Os dados obtidos foram trabalhados de forma sistemática através da ordenação, classificação e análise final do conteúdo dos artigos, seguida da etapa de tabulação dos mesmos. Por se tratar de uma pesquisa bibliográfica, este estudo não necessitou de aprovação por parte do Comitê de Ética em Pesquisa, fundamentando-se nas diretrizes e normas regulamentadoras estabelecidas nas Resoluções nº 466/2012 e 580/2018 do Conselho Nacional de Saúde do Ministério da Saúde.

\section{Resultados}

Inicialmente foram encontrados 1754 artigos, com relação às combinações entre os descritores no processo de busca, observou-se os achados estabelecidos na Tabela 1. 
Tabela 1. Disposição do número de artigos conforme arranjos dos descritores usados na pesquisa em base de dados.

\begin{tabular}{cccc}
\hline COMBINAÇÃO DE DESCRITORES & SCIELO & LILACS & 521 \\
\hline 1 E 2 & 253 & 71 & - \\
\hline 1 E 3 & 19 & 749 & - \\
\hline 2 E 3 & 88 & 33 & 15 \\
\hline 1,2 E 3 & 5 & 1374 & 15 \\
\hline Total
\end{tabular}

Legenda: Depressão (descritor 1), Idoso (descritor 2), Atenção básica (descritor 3).

Fonte: Autores. Dados obtidos nas bases de dados SCIELO, LILACS e BIREME.

Após leitura e avaliação dos trabalhos encontrados, eliminando-se a duplicidade dos escritos presentes na mesma plataforma e/ou diferentes, obteve-se a amostra final com 37 publicações, que atenderam ao conceito norteador do estudo, os critérios de inclusão e exclusão, e a análise crítica (Figura 1). Além disto, todo os dados relevantes do trabalho foram sintetizados em um quadro sinóptica para melhor visualização da catalogação (Quadro 1).

Figura 1. Processo de busca e análise dos trabalhos.

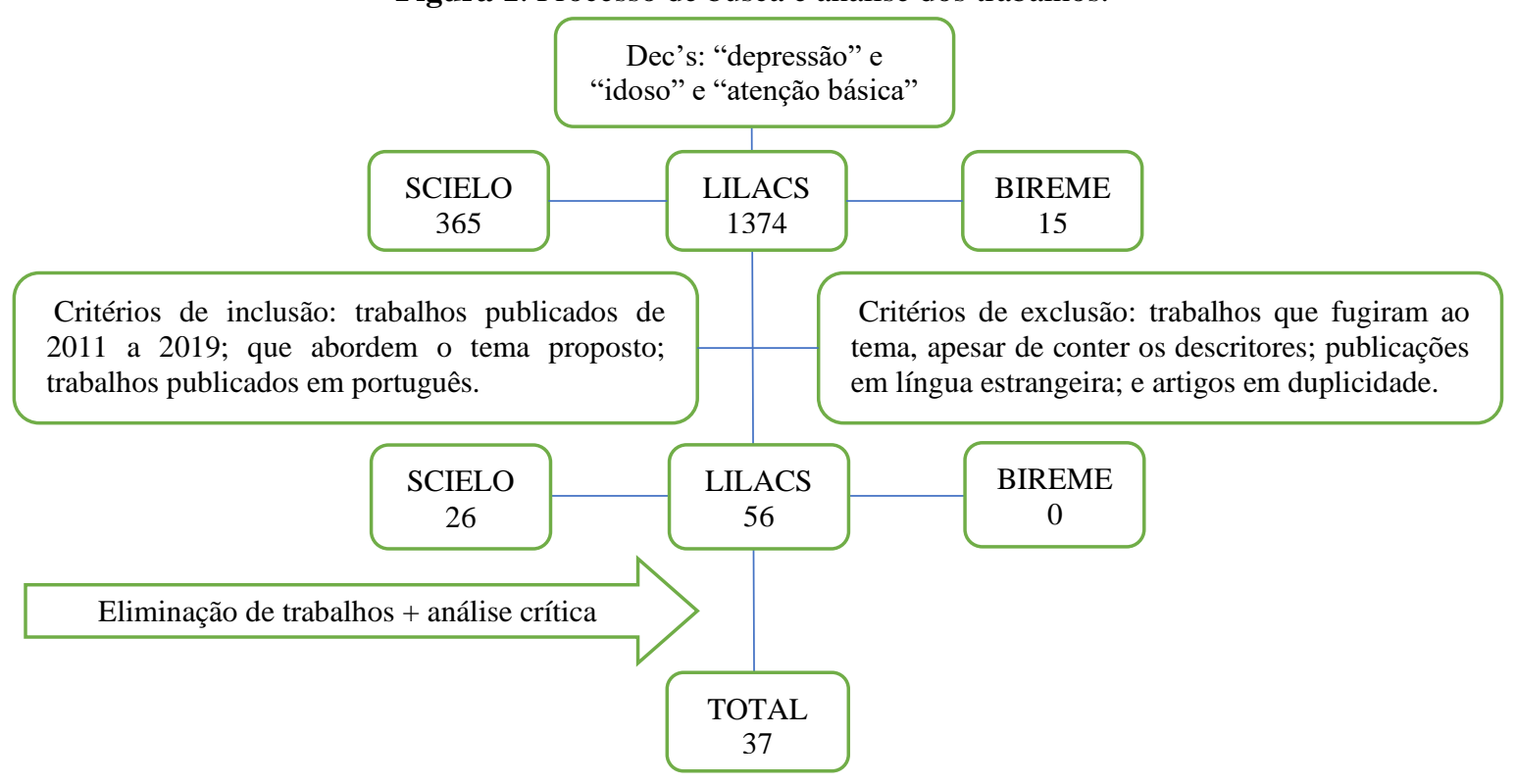

Fonte: Autores. 
Research, Society and Development, v. 10, n. 2, e23210212499, 2021

(CC BY 4.0) | ISSN 2525-3409 | DOI: http://dx.doi.org/10.33448/rsd-v10i2.12499

Quadro 1. Síntese dos estudos publicados sobre importância da atenção primária à saúde na detecção e terapêutica de sintomas depressivos em idosos

\begin{tabular}{|c|c|c|c|c|}
\hline Autor/Ano & Título & Rastreio & Terapêutica & Enfoque \\
\hline $\begin{array}{l}\text { Faria, Silva, Farias, \& Cintra, } \\
\text { (2011) }\end{array}$ & $\begin{array}{l}\text { Avaliação cognitiva de pessoas } \\
\text { idosas cadastradas na Estratégia } \\
\text { Saúde da Família: município do Sul } \\
\text { de Minas }\end{array}$ & Mini Exame do Estado Mental & Atividades físicas & $\begin{array}{l}\text { A função cognitiva no idoso pode ser } \\
\text { avaliada por vários meios, sendo o Mini } \\
\text { Mental o instrumento de avaliação mais } \\
\text { utilizado, permitindo monitorar } \\
\text { disfunções cognitivas associadas a outras } \\
\text { doenças, bem como mudanças sutis; além } \\
\text { de investigar a prevalência e a incidência } \\
\text { de processos demenciais. }\end{array}$ \\
\hline Lino (2011) & $\begin{array}{l}\text { Triagem de problemas de saúde de } \\
\text { idosos na Atenção Primária com um } \\
\text { instrumento multidimensional }\end{array}$ & $\begin{array}{l}\text { Mini Exame do Estado Mental; } \\
\text { Escala de Katz; Avaliação } \\
\text { Geriátrica Ampla. }\end{array}$ & Apoio social & $\begin{array}{l}\text { Adota medidas para a adequação da } \\
\text { atenção aos idosos e o rastreamento de } \\
\text { problemas de saúde por meio de } \\
\text { ferramentas padronizadas, possibilitando } \\
\text { a identificação de pacientes que } \\
\text { necessitam de intervenções específicas e } \\
\text { individualizadas, de acordo com o } \\
\text { problema apresentado, destacando a } \\
\text { importância da atenção primária à saúde } \\
\text { na detecção destes problemas.. }\end{array}$ \\
\hline Beltrão et al. (2011) & $\begin{array}{l}\text { Sintomatologia da } \\
\begin{array}{l}\text { depressão em } \\
\text { idosos atendidos em unidades } \\
\text { básicas de saúde }\end{array}\end{array}$ & Escala de Depressão Geriátrica & Cuidado humanizado & $\begin{array}{l}\text { Estudo exploratório realizado em } \\
\text { Unidades Básicas de Saúde do município } \\
\text { de João Pessoa com o objetivo de } \\
\text { conhecer a prevalência da sintomatologia } \\
\text { depressiva em idosos. Além de também } \\
\text { destacar a importância da atenção } \\
\text { primária à saúde nesta área. }\end{array}$ \\
\hline $\begin{array}{l}\text { Alvarenga, Oliveira, \& Faccenda } \\
\text { (2012) }\end{array}$ & $\begin{array}{l}\text { Sintomas depressivos em idosos: } \\
\text { análise dos itens da Escala de } \\
\text { Depressão Geriátrica }\end{array}$ & Escala de Depressão Geriátrica & $\begin{array}{l}\text { Atividades físicas, } \\
\text { recreativas e culturais }\end{array}$ & $\begin{array}{l}\text { Estudo de delineamento transversal com } \\
503 \text { idosos assistidos pela Estratégia } \\
\text { Saúde da Família, em Dourados, MS. } \\
\text { Analisou-se a resposta de pacientes com } \\
\text { apatia, desesperança, desmotivação e } \\
\text { isolamento submetidos à Escala de } \\
\text { Depressão Geriátrica. }\end{array}$ \\
\hline Oliveira et al. (2012) & $\begin{array}{l}\text { Sintomatologia de depressão } \\
\text { autorreferida por idosos que vivem } \\
\text { em comunidade }\end{array}$ & $\begin{array}{l}\text { Escala de Depressão Geriátrica } \\
\text { Abreviada }\end{array}$ & - & $\begin{array}{l}\text { Avalia a sintomatologia da depressão } \\
\text { autorreferida por idosos, considerando } \\
\text { variáveis }\end{array}$ \\
\hline
\end{tabular}


Research, Society and Development, v. 10, n. 2, e23210212499, 2021

(CC BY 4.0) | ISSN 2525-3409 | DOI: http://dx.doi.org/10.33448/rsd-v10i2.12499

\begin{tabular}{|c|c|c|c|c|}
\hline & & & & sociodemográficas. \\
\hline Molina et al. (2012) & $\begin{array}{l}\text { Prevalência de depressão em } \\
\text { usuários de unidades de Atenção } \\
\text { Primária }\end{array}$ & - & $\begin{array}{l}\text { Intervenções psicossociais } \\
\text { e farmacológicas }\end{array}$ & $\begin{array}{l}\text { Mostra como o diagnóstico de depressão } \\
\text { costuma ser prejudicado pela presença } \\
\text { frequente de comorbidades, pela } \\
\text { dificuldade da equipe de saúde em } \\
\text { reconhecê-la e pela falta de atenção à } \\
\text { saúde mental no sistema de saúde } \\
\text { primário. }\end{array}$ \\
\hline Rodrigues et al. (2012) & $\begin{array}{l}\text { Qualidade de vida de idosos com } \\
\text { indicativo de depressão: implicações } \\
\text { para a enfermagem }\end{array}$ & $\begin{array}{l}\text { Mini Exame de Estado Mental; } \\
\text { Escala de Depressão Geriátrica } \\
\text { Abreviada }\end{array}$ & $\begin{array}{l}\text { Farmacológico; } \\
\text { Intervenções psicossociais }\end{array}$ & $\begin{array}{l}\text { Estudo descritivo, transversal e } \\
\text { observacional que objetivou caracterizar } \\
187 \text { idosos com indicativo de depressão, } \\
\text { residentes na zona rural de Uberaba, } \\
\text { segundo variáveis sociodemográficas e } \\
\text { econômicas e mensurar a qualidade de } \\
\text { vida desses idosos. }\end{array}$ \\
\hline $\begin{array}{l}\text { Borges, Benedetti, Xavier, \& d'Orsi } \\
\text { (2012) }\end{array}$ & $\begin{array}{l}\text { Fatores associados aos sintomas } \\
\text { depressivos em idosos: estudo } \\
\text { EpiFloripa }\end{array}$ & $\begin{array}{l}\text { Escala de Depressão Geriátrica } \\
\text { Abreviada }\end{array}$ & Grupos de convivência & $\begin{array}{l}\text { Mostra a situação clínica adversa da } \\
\text { população avaliada, desvantagem } \\
\text { socioeconômica e relata que pouca } \\
\text { atividade social e sexual influencia na } \\
\text { associação dos sintomas depressivos em } \\
\text { idosos. }\end{array}$ \\
\hline Santos (2013) & $\begin{array}{l}\text { Avaliação do déficit cognitivo e sua } \\
\text { relação com características } \\
\text { sociodemográficas, com condições } \\
\text { de saúde e com o estilo de vida de } \\
\text { pessoas idosas atendidas na Atenção } \\
\text { Básica no município de Jacareí, São } \\
\text { Paulo. }\end{array}$ & $\begin{array}{l}\text { Mini Exame do Estado Mental; } \\
\text { Escala Geriátrica de } \\
\text { Depressão; Escalas de } \text { Katz e } \\
\text { Lawton. }\end{array}$ & $\begin{array}{l}\text { Farmacológico e/ou ações } \\
\text { de reabilitação } \\
\text { neuropsicológica }\end{array}$ & $\begin{array}{l}\text { Analisa a prevalência do déficit cognitivo } \\
\text { e a sua associação com outras condições } \\
\text { de saúde e com fatores sociodemográficos } \\
\text { em idosos, considerando-se o intenso } \\
\text { crescimento da proporção de idosos que } \\
\text { resulta em um grau elevado de } \\
\text { incapacitação nessa população. Destaca o } \\
\text { trabalho na atenção primária à saúde para } \\
\text { o reconhecimento destas alteracões. }\end{array}$ \\
\hline $\begin{array}{l}\text { Holz, Nunes, Thumé, Lange, \& } \\
\text { Facchini (2013) }\end{array}$ & $\begin{array}{l}\text { Prevalência de déficit cognitivo e } \\
\text { fatores associados entre idosos de } \\
\text { Bagé, Rio Grande do Sul, Brasil }\end{array}$ & Mini Exame do Estado Mental & Ações de saúde mental & $\begin{array}{l}\text { Objetivou identificar a prevalência e } \\
\text { fatores associados ao déficit cognitivo em } \\
\text { idosos residentes na área de abrangência } \\
\text { dos serviços de atenção básica em saúde. }\end{array}$ \\
\hline $\begin{array}{l}\text { Silva, Pereira, Guimarães, Perrelli, } \\
\text { \& Santos (2014) }\end{array}$ & $\begin{array}{l}\text { Depressão: conhecimento de idosos } \\
\text { atendidos em unidades de saúde da } \\
\text { família no município de Limoeiro- } \\
\text { PE }\end{array}$ & Entrevistas dirigidas ao idosos & Ações de saúde mental & $\begin{array}{l}\text { Evidenciou a necessidade de inserir } \\
\text { efetivamente as ações de prevenção ao } \\
\text { adoecimento mental, especificamente a } \\
\text { depressão, nas unidades de saúde da }\end{array}$ \\
\hline
\end{tabular}


Research, Society and Development, v. 10, n. 2, e23210212499, 2021

(CC BY 4.0) | ISSN 2525-3409 | DOI: http://dx.doi.org/10.33448/rsd-v10i2.12499

\begin{tabular}{|c|c|c|c|c|}
\hline & & & & $\begin{array}{l}\text { família, para que se possa desenvolver um } \\
\text { cuidado integral ao usuário idoso, à } \\
\text { família e comunidade. }\end{array}$ \\
\hline Lima et al. (2014) & $\begin{array}{l}\text { Humanização na atenção básica de } \\
\text { saúde na percepção de idosos }\end{array}$ & Entrevistas dirigidas aos idosos & Cuidado humanizado & $\begin{array}{l}\text { Analisa a percepção do idoso quanto ao } \\
\text { cuidado humanizado na atenção básica de } \\
\text { saúde. }\end{array}$ \\
\hline $\begin{array}{l}\text { Martins, D'Avila, Hilgert, \& Hugo } \\
\text { (2014) }\end{array}$ & $\begin{array}{l}\text { Atenção primária à saúde voltada as } \\
\text { necessidades dos idosos: da teoria à } \\
\text { prática }\end{array}$ & $\begin{array}{l}\text { Mini Exame do Estado Mental; } \\
\text { Escala de Depressão Geriátrica } \\
\text { Abreviada }\end{array}$ & Intervenções psicossociais & $\begin{array}{l}\text { Estabelece uma confrontação entre teoria } \\
\text { e prática no que tange ao cuidado com a } \\
\text { saúde de pessoas idosas, na atenção } \\
\text { primária à saúde. }\end{array}$ \\
\hline $\begin{array}{l}\text { Nogueira, Rubin, Giacobbo, } \\
\text { Gomes, \& Cataldo Neto (2014) }\end{array}$ & $\begin{array}{l}\text { Rastreamento de sintomas } \\
\text { depressivos em idosos na Estratégia } \\
\text { Saúde da Família, Porto Alegre }\end{array}$ & $\begin{array}{l}\text { Escala de Depressão Geriátrica } \\
\text { Abreviada }\end{array}$ & - & $\begin{array}{l}\text { Evidenciou similaridade com outros } \\
\text { estudos que também constataram que a } \\
\text { depressão se associa à baixa escolaridade, } \\
\text { ao sexo feminino e à pior autopercepção } \\
\text { de saúde. }\end{array}$ \\
\hline Bretanha et al. (2015) & $\begin{array}{l}\text { Sintomas depressivos em idosos } \\
\text { residentes em áreas de abrangência } \\
\text { das Unidades Básicas de Saúde da } \\
\text { zona urbana de Bagé, RS }\end{array}$ & $\begin{array}{l}\text { Escala de Depressão Geriátrica } \\
\text { Abreviada; Escalas de Katz e } \\
\text { de Lawton e Brody. }\end{array}$ & $\begin{array}{l}\text { Intervenções } \\
\text { psicossociais; } \\
\text { humanizado }\end{array}$ & $\begin{array}{l}\text { Identifica a prevalência de sintomas } \\
\text { depressivos e os fatores associados na } \\
\text { população idosa, mostrando a necessidade } \\
\text { de investimento em ações de prevenção, } \\
\text { especialmente na atenção primária à } \\
\text { saúde, atentando para a necessidade de } \\
\text { práticas que promovam o envelhecimento } \\
\text { ativo. }\end{array}$ \\
\hline $\begin{array}{l}\text { Frade, Barbosa, Cardoso, \& Nunes } \\
\text { (2015) }\end{array}$ & $\begin{array}{l}\text { Depressão no idoso: sintomas em } \\
\text { indivíduos institucionalizados e não- } \\
\text { institucionalizados }\end{array}$ & Escala de Depressão Geriátrica & $\begin{array}{l}\text { Exercícios } \\
\text { Intervenções } \\
\text { psicossociais; } \\
\text { humanizado }\end{array}$ & $\begin{array}{l}\text { As perturbações psiquiátricas no idoso } \\
\text { são comuns e responsáveis pela perda de } \\
\text { autonomia, pelo agravamento de quadros } \\
\text { patológicos preexistentes e pela } \\
\text { negligência no autocuidado; além de } \\
\text { estudar a associação entre a } \\
\text { institucionalização e a presença de } \\
\text { depressão em idosos. }\end{array}$ \\
\hline Lentsck et al. (2015) & $\begin{array}{l}\text { Prevalência de sintomas depressivos } \\
\text { e sinas de demência em idosos na } \\
\text { comunidade }\end{array}$ & Brazil Old Age Schedule & $\begin{array}{l}\text { Intervenções } \\
\text { psicossociais; } \\
\text { humanizado }\end{array}$ & $\begin{array}{l}\text { Estudo transversal que analisou a } \\
\text { prevalência de sintomas depressivos, } \\
\text { sinais de demência e fatores associados } \\
\text { em idosos residentes em município do sul } \\
\text { do Brasil. Foi realizado inquérito } \\
\text { domiciliar a uma amostra de } 359 \text { idosos } \\
\text { cadastrados nas Unidades Básicas de }\end{array}$ \\
\hline
\end{tabular}


Research, Society and Development, v. 10, n. 2, e23210212499, 2021

(CC BY 4.0) | ISSN 2525-3409 | DOI: http://dx.doi.org/10.33448/rsd-v10i2.12499

\begin{tabular}{|c|c|c|c|c|}
\hline & & & & $\begin{array}{l}\text { Saúde, enfatizando o trabalho da atenção } \\
\text { primária à saúde quanto ao diagnóstico e } \\
\text { prevenção. }\end{array}$ \\
\hline Ramos et al. (2015) & $\begin{array}{l}\text { Prevalência de sintomas depressivos } \\
\text { e fatores associados em idosos no } \\
\text { norte de Minas Gerais: um estudo de } \\
\text { base populacional }\end{array}$ & $\begin{array}{l}\text { Escala Geriátrica de Depressão } \\
\text { Abreviada }\end{array}$ & - & $\begin{array}{l}\text { Avaliação da prevalência dos fatores } \\
\text { associados aos sintomas depressivos em } \\
\text { idosos não institucionalizados. }\end{array}$ \\
\hline Rodrigues et al. (2015) & $\begin{array}{l}\text { Qualidade de vida, indicativo de } \\
\text { depressão e número de morbidades } \\
\text { de idosos da zona rural }\end{array}$ & $\begin{array}{l}\text { Mini Exame do Estado Mental; } \\
\text { Escala de Depressão Geriátrica } \\
\text { Abreviada }\end{array}$ & $\begin{array}{l}\text { Intervenções } \\
\text { psicossociais; } \quad \text { Cuidado } \\
\text { humanizado }\end{array}$ & $\begin{array}{l}\text { Objetivou-se caracterizar os idosos com } \\
\text { indicativo de depressão, mensurar e } \\
\text { correlacionar os escores de qualidade de } \\
\text { vida com o número de morbidades. }\end{array}$ \\
\hline Magalhães et al. (2016) & $\begin{array}{l}\text { Depressão em idosos na estratégia } \\
\text { saúde da família: uma contribuição } \\
\text { para a atenção primária. }\end{array}$ & $\begin{array}{l}\text { Escala de Depressão Geriátrica } \\
\text { (EDG) }\end{array}$ & $\begin{array}{l}\text { Farmacológico; } \\
\text { Intervenções psicossociais }\end{array}$ & $\begin{array}{l}\text { Estima a prevalência de depressão em } \\
\text { idosos em uma unidade básica de saúde, } \\
\text { identifica os quadros de depressão na } \\
\text { população de idosos que realiza } \\
\text { acompanhamento nesta UBS e o uso de } \\
\text { medicação para tratamento dos } \\
\text { transtornos. }\end{array}$ \\
\hline Lino et al. (2016) & $\begin{array}{l}\text { Rastreamento de problemas de } \\
\text { idosos na atenção primária e } \\
\text { proposta de roteiro de triagem com } \\
\text { uma abordagem multidimensional }\end{array}$ & $\begin{array}{l}\text { Mini Exame do Estado Mental, } \\
\text { Escala de Katz; Avaliação } \\
\text { Geriátrica Ampla }\end{array}$ & - & $\begin{array}{l}\text { Mostra como o envelhecimento } \\
\text { frequentemente acarreta alterações } \\
\text { funcionais insidiosas, demonstra como } \\
\text { instrumentos de rastreamento de } \\
\text { condições desencadeadoras de declínio } \\
\text { funcional permitem intervenções } \\
\text { precoces, de maior impacto. }\end{array}$ \\
\hline Gullich et al. (2016) & $\begin{array}{l}\text { Depressão entre idosos: um estudo } \\
\text { de base populacional no Sul do } \\
\text { Brasil }\end{array}$ & $\begin{array}{l}\text { Escala de Depressão Geriátrica } \\
\text { Abreviada }\end{array}$ & $\begin{array}{lr}\text { Exercícios } & \text { físicos; } \\
\text { Intervenções } & \\
\text { psicossociais; } & \text { Cuidado } \\
\text { humanizado } & \end{array}$ & $\begin{array}{l}\text { Este estudo mediu a prevalência e } \\
\text { identificou fatores associados à ocorrência } \\
\text { de depressão entre idosos. Aplicou-se } \\
\text { questionário domiciliar a todos aqueles } \\
\text { com } 60 \text { anos ou mais. O desfecho foi } \\
\text { avaliado pela Escala de Depressão } \\
\text { Geriátrica Reduzida (EDG-15). }\end{array}$ \\
\hline Hellwig et al. (2016) & $\begin{array}{l}\text { Sintomas depressivos em } \text { idosos: } \\
\text { estudo transversal de base } \\
\text { populacional }\end{array}$ & $\begin{array}{l}\text { Escala de Depressão Geriátrica } \\
\text { Abreviada }\end{array}$ & & $\begin{array}{l}\text { Este estudo teve como objetivo estimar a } \\
\text { ocorrencia de sintomas depressivos em } \\
\text { indivíduos com } 60 \text { anos ou mais e } \\
\text { identificar os seus fatores associados. }\end{array}$ \\
\hline
\end{tabular}


Research, Society and Development, v. 10, n. 2, e23210212499, 2021

(CC BY 4.0) | ISSN 2525-3409 | DOI: http://dx.doi.org/10.33448/rsd-v10i2.12499

\begin{tabular}{|c|c|c|c|c|}
\hline & & & - & \\
\hline $\begin{array}{l}\text { Matias, Fonsêca, Gomes, \& Matos } \\
\text { (2016) }\end{array}$ & $\begin{array}{l}\text { Indicadores de depressão em idosos } \\
\text { e os diferentes métodos de } \\
\text { rastreamento }\end{array}$ & Escala de Depressão Geriátrica & - & $\begin{array}{l}\text { Determina a prevalência dos sintomas } \\
\text { depressivos entre idosos e correlacionar a } \\
\text { concordância dos métodos de } \\
\text { rastreamentos utilizados. }\end{array}$ \\
\hline Piani et al. (2016) & $\begin{array}{l}\text { Prevalência de sintomas depressivos } \\
\text { em idosas de um Centro de } \\
\text { Referência de Atenção ao Idoso no } \\
\text { município de Passo Fundo, Rio } \\
\text { Grande do Sul }\end{array}$ & Escala de Depressão Geriátrica & Intervenções psicossociais & $\begin{array}{l}\text { Mostra que mudanças psicológicas, } \\
\text { sociais e biológicas, ao longo da vida, } \\
\text { podem tornar o idoso vulnerável a } \\
\text { desenvolver determinadas patologias. }\end{array}$ \\
\hline Previato et al. (2016) & $\begin{array}{l}\text { Características multidimensionais de } \\
\text { saúde de idosos com sintomas } \\
\text { depressivos }\end{array}$ & \begin{tabular}{l}
\multicolumn{3}{l}{ Escala de Depressão Geriátrica } \\
Abreviada; Mini Exame do \\
Estado Mental; Escala de \\
Lawton e Brody; Índice de \\
Katz; $\quad$ Protocolo \\
Vulnerabilidade do Idoso
\end{tabular} & $\begin{array}{l}\text { Farmacológico; } \\
\text { Intervenções } \\
\text { psicossociais; Cuidado } \\
\text { humanizado }\end{array}$ & $\begin{array}{l}\text { Analisou as características } \\
\text { multidimensionais de saúde de idosos } \\
\text { com sintomas depressivos. }\end{array}$ \\
\hline Souza et al. (2017) & $\begin{array}{l}\text { Avaliação da assistência à pessoa } \\
\text { idosa na atenção primária à saúde: } \\
\text { perspectiva de usuários. }\end{array}$ & Estudo exploratório descritivo & $\begin{array}{l}\text { Atividades recreativas, } \\
\text { culturais e educativas }\end{array}$ & $\begin{array}{l}\text { Avalia a importância de uma assistência } \\
\text { bem feita por parte de uma equipe } \\
\text { multidisciplinar da atenção básica. }\end{array}$ \\
\hline Medeiros et al. (2017) & $\begin{array}{l}\text { O aumento do contingente } \\
\text { populacional de idosos no Brasil e a } \\
\text { atenção primária à saúde: uma } \\
\text { revisão de literatura }\end{array}$ & - & - & $\begin{array}{l}\text { Discutiu o aumento do contingente } \\
\text { populacional de idosos no Brasil e a sua } \\
\text { repercussão na Atenção Primária à Saúde. }\end{array}$ \\
\hline Pagliuca et al. (2017) & $\begin{array}{l}\text { Acesso de idosos às unidades de } \\
\text { atenção primária à saúde }\end{array}$ & 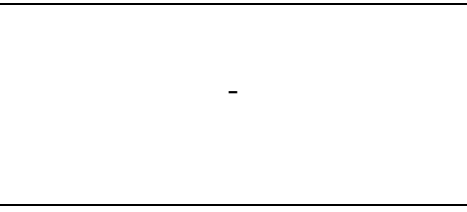 & - & $\begin{array}{l}\text { Apresenta um panorama sobre as } \\
\text { condições insatisfatórias de acesso às } \\
\text { unidades de saúde, demonstrando que os } \\
\text { serviços na atenção primária não têm } \\
\text { funcionado adequadamente. }\end{array}$ \\
\hline Rêgo et al. (2017) & $\begin{array}{l}\text { Fatores associados ao atendimento a } \\
\text { idosos por condições sensíveis à } \\
\text { Atenção Primária à Saúde }\end{array}$ & Mini Exame de Estado Mental & - & $\begin{array}{l}\text { Analisa fatores associados ao atendimento } \\
\text { a idosos em Unidade de Pronto } \\
\text { Atendimento por condições sensíveis à } \\
\text { Atenção Primária à Saúde. }\end{array}$ \\
\hline Silva et al. (2017) & $\begin{array}{l}\text { Doenças crônicas não transmissíveis } \\
\text { e fatores sociodemográficos } \\
\text { associados a sintomas de depressão } \\
\text { em idosos }\end{array}$ & Escala de Depressão Geriátrica & Ações de saúde mental & $\begin{array}{l}\text { Mostrou a associação das doenças } \\
\text { crônicas não transmissíveis e fatores } \\
\text { sociodemográficos com sintomas de } \\
\text { depressão em idosos. }\end{array}$ \\
\hline
\end{tabular}


Research, Society and Development, v. 10, n. 2, e23210212499, 2021

(CC BY 4.0) | ISSN 2525-3409 | DOI: http://dx.doi.org/10.33448/rsd-v10i2.12499

\begin{tabular}{|c|c|c|c|c|}
\hline Soares et al. (2017) & $\begin{array}{l}\text { Associação entre depressão e } \\
\text { qualidade de vida em idosos: atenção } \\
\text { primária à saúde }\end{array}$ & Mini Exame do Estado Mental & Ações de saúde mental & $\begin{array}{l}\text { Avalia associação entre depressão e } \\
\text { qualidade de vida em idosos. }\end{array}$ \\
\hline Apóstolo et al. (2018) & $\begin{array}{l}\text { Capacidade de rastreio da Escala de } \\
\text { Depressão Geriátrica com } 10 \text { e } 5 \\
\text { itens }\end{array}$ & $\begin{array}{l}\text { Escala de Depressão Geriátrica } \\
\text { Abreviada }\end{array}$ & - & $\begin{array}{l}\text { Avalia capacidade de rastreio de } \\
\text { depressão por meio das escalas de } \\
\text { depressão geriátrica abreviada com } 10 \text { e } 5 \\
\text { itens, com o objetivo de reduzir as } \\
\text { consequências gravosas resultantes da } \\
\text { mesma. }\end{array}$ \\
\hline Gil et al. (2018) & $\begin{array}{l}\text { Eficácia da reminiscência na } \\
\text { cognição, sintomas depressivos e } \\
\text { qualidade de vida em idosos: } \\
\text { protocolo de revisão sistemática }\end{array}$ & \begin{tabular}{llr} 
Escala de & \multicolumn{2}{c}{ Depressão } \\
Geriátrica; Mini & Exame do \\
Estado Mental &
\end{tabular} & Terapia de reminiscência & $\begin{array}{l}\text { As intervenções não-farmacológicas } \\
\text { apresentam-se como valiosas estratégias } \\
\text { terapêuticas dirigidas às pessoas com } \\
\text { compromisso cognitivo, destacando-se na } \\
\text { literatura a terapia de reminiscência. }\end{array}$ \\
\hline Abrantes et al. (2019) & $\begin{array}{l}\text { Sintomas depressivos em idosos na } \\
\text { atenção básica à saúde }\end{array}$ & $\begin{array}{l}\text { Escala de Depressão Geriátrica } \\
\text { Abreviada }\end{array}$ & Ações de saúde mental & $\begin{array}{l}\text { Identificar sintomas depressivos em } \\
\text { idosos atendidos na atenção básica. }\end{array}$ \\
\hline $\begin{array}{l}\text { Pereira, Lima, Salgueiro, Carvalho } \\
\text { (2019) }\end{array}$ & $\begin{array}{l}\text { Atuação da enfermagem frente à } \\
\text { depressão na população idosa }\end{array}$ & - & Psicológico & $\begin{array}{l}\text { Identificar estudos sobre a atuação da } \\
\text { enfermagem frente à depressão nos } \\
\text { idosos. }\end{array}$ \\
\hline Uchoa et al. (2019) & $\begin{array}{l}\text { Fatores associados a sintomas } \\
\text { depressivos e capacidade } \\
\text { em iduncional } \\
\end{array}$ & 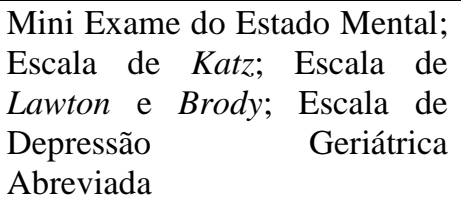 & - & $\begin{array}{l}\text { Identificar a prevalência e fatores } \\
\text { associados a sintomas depressivos e } \\
\text { capacidade funcional em idosos }\end{array}$ \\
\hline
\end{tabular}

Fonte: Autores. 
Entre os 37 trabalhos avaliados, observou-se que 25 (67,56\%) deles demonstraram prevalência de sintomas depressivos nos idosos do sexo feminino, indivíduos que já perderam seus companheiros, portadores de doenças crônicas, aposentados e pacientes polimedicamentosos.

Em relação às comorbidades na senescência, 26 (70,27\%) dos artigos demonstraram que os idosos com sintomas depressivos possuem tendência grave à depressão, e apresentam maior vulnerabilidade a ter declínio cognitivo, perda de autonomia e restrição ao leito.

Identificou-se, também, os instrumentos práticos mais utilizados para detecção dos sintomas depressivos de forma precoce (Quadro 1). O instrumento mais descrito na literatura ao longo dos anos foi a Escala de Depressão Geriátrica, sendo evidenciado frequência de citação em 23 publicações; seguido do Mini Exame do Estado Mental, Escala de Brody e Lawton, com 13, 06 e 04 dos trabalhos, respectivamente, que possuem essa informação.

Observou-se predomínio no uso dos métodos não farmacológicos e que contemplassem o ser em seu estado biopsicossocial, sendo assim práticas como atividades físicas, recreativas, culturais e participação em grupos de convivência foram recomendadas em $23(62,16 \%)$ dos artigos; seguido de apenas 04 (10,81\%) trabalhos recomendando uso de métodos farmacológicos. Evidenciou-se, ainda, a importância da atenção primária na prevenção, diagnóstico e cuidado dos idosos em $16(43,24 \%)$ trabalhos, com descrição de dificuldades, manejo e assertivas na gestão da problemática.

\section{Discussão}

Com o envelhecimento populacional sendo uma realidade cada vez mais frequente (Medeiros et al., 2017), evidenciou-se que idosos com sintomas depressivos são fortes candidatos a terem depressão devido a maior vulnerabilidade, declínio cognitivo, perda de autonomia e restrição ao leito (Santos, 2013; Holz et al., 2013; Piani et al., 2016; Gil et al., 2018); sintomas, por vezes, confundidos com sentimentos inerentes a idade (Previato et al., 2016; Lino et al., 2016). Essa constatação suscita a necessidade de se intervir precocemente, no intuito de evitar maior agravamento depressivo e perda da qualidade de vida (Rodrigues et al., 2012; Rodrigues et al., 2015; Soares et al., 2017; Abrantes et al., 2019).

A maioria das alterações têm início insidioso, mas pode ser diagnosticada por instrumentos de rastreamento de condições desencadeadoras de declínio funcional (Santos, 2013), como Mini Exame do Estado Mental, Escala de Depressão Geriátrica, Escala Katz; Escala de Lawton e Escala Brody. O diagnóstico precoce permite maiores possibilidades de planejamento das ações na área da saúde e atende às proposições da Organização Mundial de Saúde e do Ministério da Saúde do Brasil para a triagem de idosos (Lino et al., 2016), porém a falta de padronização de uma escala nacional pode dificultar os rastreios.

A atenção primária à saúde deve estar preparada para o recebimento e implementação desses mecanismos de rastreios na população (Borges et al., 2013; Souza et al., 2017; Abrantes et al., 2019), visto que é o primeiro serviço buscado para prosseguimento da assistência à saúde por meio do sistema público, e relatado em cerca de $43 \%$ dos trabalhos. A escolha do melhor método deve partir do profissional de saúde capacitado (Matias et al., 2016) para identificação da problemática e avaliação do nível depressivo dos usuários (Nogueira et al., 2014; Lino et al., 2016), utilizando assim instrumentos validados.

Dentre os mecanismos mais evidenciadas nos artigos e utilizados na prática clínica das unidades de atenção básica, a Escala de Depressão Geriátrica se mostrou mais presente nas avaliações (Quadro 1). Esse instrumento possui 30 questionamentos que devem ser respondidos pelo paciente para melhor avaliação. Versões reduzidas dessa escala também são aplicadas, com 15, 10 e 5 questões (Oliveira et al., 2012; Rodrigues et al., 2012; Borges et al., 2012; Martins et al., 2014; Nogueira et al., 2014; Bretanha et al., 2015; Rodrigues et al., 2015; Gullich et al., 2016; Hellwig et al., 2016; Previato et al., 2016; Apóstolo et al., 2018; Abrantes et al., 2019; Uchoa et al., 2019), mas devem ser medidas com ressalvas no intuito de 
Research, Society and Development, v. 10, n. 2, e23210212499, 2021

(CC BY 4.0) | ISSN 2525-3409 | DOI: http://dx.doi.org/10.33448/rsd-v10i2.12499

reduzir falsos positivos (Apóstolo et al., 2018).

O Mini Exame do Estado Mental e a Avaliação Geriátrica Ampla se apresentam como bons métodos de rastreamento, que permitem monitorar disfunções cognitivas associadas a outras doenças, bem como mudanças sutis, além de investigar a prevalência e a incidência de processos demenciais, sendo bem evidenciado nos trabalhos avaliados (Faria et al., 2011; Lino, 2011; Rodrigues et al., 2012; Santos, 2013; Holz et al., 2013; Martins et al., 2014; Rodrigues et al., 2015; Lino et al., 2016; Previato et al., 2016; Rêgo et al., 2017; Soares et al., 2017; Gil et al.,2018; Uchoa et al., 2019). Ambos os testes se mostram adequados para implementação na atenção primária à saúde, porém devido ao longo tempo para aplicação e alta demanda por atendimentos, elas devem ser consideradas apenas a pacientes com maior risco de incapacidade.

A escala de Lawton e Brody para capacidade funcional para atividades instrumentais de vida diária, e o Índice de Katz, para capacidade funcional para atividades básicas de vida diária, apresentam-se como instrumentos simples, de baixo custo, capazes de identificar os riscos e a dimensão de adoecimento de forma precoce a partir da análise do cotidiano da população idosa (Lino, 2011; Santos, 2013; Bretanha et al., 2015; Lino et al., 2016; Previato et al., 2016; Uchoa et al., 2019). Fato importante quando socialmente se admite redução e se atribui incapacidade do indivíduo ao adentrar na terceira idade para execução de fazeres básicos, servindo de medidor para intervenção familiar e acompanhamento mais próximo daqueles que necessitam.

O método Brazil Old Age Schedule se apresenta como instrumento multidimensional que avalia aspectos de vida do idoso, medindo também impactos nas atividades do dia a dia, como situação social, econômica, física e mental. Lentsk et al. (2015) abordam que o método se constitui como ferramenta interessante a ser utilizada na atenção básica porque pode ser aplicada por outros profissionais, não necessitando obrigatoriamente do médico; constatação que evidencia a possibilidade de maior cobertura do procedimento de rastreio pela equipe de saúde aos usuários do serviço público.

Em relação ao Protocolo de Identificação do Idoso Vulnerável, que também avalia a vulnerabilidade do idoso, segundo a catalogação e avaliação dos dados sintetizados na Quadro 1, é um método confiável e validado (Previato et al., 2016), que tem sido utilizado como um instrumento de rastreio em pacientes oncológicos. Apesar do seu emprego ser relatado mais em idosos com câncer, possui plena capacidade de atender a demanda das outras situações clínicas da população, em especial na atenção primária.

Feita a identificação dos perfis dos usuários em relação aos sintomas depressivos, por meio dos métodos previamente elencados, açães de prevenção e tratamentos precisam ser efetivados. Os trabalhos avaliados demonstraram preocupação e predomínio por tratamentos não farmacológicos e que contemplavam o ser em seu estado biopsicossocial (Soares et al., 2017; Gil et al., 2018; Pereira et al., 2019). Sabe-se que a população idosa, foco desta revisão, é marcada pelo perfil polimedicamentoso (Borges et al., 2013) devido a uma grande incidência de doenças crônicas não transmissíveis (Silva et al., 2017), e o cuidado com a promoção do uso racional de medicamento observado se materializa como uma mudança gradual de educação em saúde na integralização do cuidado multiprofissional.

Nota-se, assim, a importância da atenção primária à saúde na detecção e terapêutica de sintomas depressivos na população idosa. Os profissionais da saúde precisam estar aptos para aplicação e compreensão do melhor método, individualizando o paciente à usabilidade da ferramenta que melhor se enquadra ao perfil do indivíduo, além de promoverem ações de saúde por meio de tratamentos assistidos, sejam não farmacológicos e/ou farmacológicos.

\section{Conclusão}

Foi possível identificar os principais sintomas de depressão em idosos atendidos em unidades básicas de saúde elencados nos trabalhos avaliados, como declínio cognitivo e perda da autonomia por meio da utilização de técnicas de 
Research, Society and Development, v. 10, n. 2, e23210212499, 2021

(CC BY 4.0) | ISSN 2525-3409 | DOI: http://dx.doi.org/10.33448/rsd-v10i2.12499

rastreio, sendo a Escala de Depressão Geriátrica a mais empregada.

A partir desta identificação da gravidade dos casos, medidas terapêuticas diversas - medicamentosas e/ou não medicamentosas - foram avaliadas e empregadas. Esses mecanismos atendem recomendações nacionais e internacionais, reforçando a importância da assistência primária à saúde como mais um veículo de promoção a melhoria da qualidade de vida, implicando diretamente na chance de uma velhice mais saudável.

Em virtude da importância da temática avaliada, sugere-se que periodicamente novas pesquisas venham a público com o intuito de atualização constante dos levantamentos pontuados, favorecendo difusão técnico-científica, ações de políticas públicas e, ainda, lançar base para uma possível padronização dos métodos de rastreio, com foco na facilidade da empregabilidade e manutenção da precisão dos resultados.

\section{Referências}

Abrantes, G. G. D., Souza, G. G., Cunha, N. M., Rocha, H. N. B. D., Silva, A. O., \& Vasconcelos, S. C. (2019). Sintomas depressivos em idosos na atenção básica à saúde. Revista Brasileira de Geriatria e Gerontologia, 22(4).

Alvarenga, M. R. M., Oliveira, M. A. D. C., \& Faccenda, O. (2012). Sintomas depressivos em idosos: análise dos itens da Escala de Depressão Geriátrica. Acta Paulista de Enfermagem, 25(4), 497-503.

Apóstolo, J. L. A., Bobrowicz-Campos, E. M., Reis, I. A. C. D., Henriques, S. J., \& Correia, C. A. V. (2018). Capacidade de rastreio da Escala de Depressão Geriátrica com 10 e 5 itens. Revista de Enfermagem Referência, (16), 29-40.

Beltrão, I. N., Silva, L. M., Alves, M. D. S. C. F., Moreira, M. A. S. P., Mendes, F., \& Targino, R. R. B. (2011). Sintomatologia da depressão em idosos atendidos em unidades básicas de saúde. Revista de Pesquisa Cuidado é Fundamental Online, 3, 1-8.

Borges, L. J., Benedetti, T. R. B., Xavier, A. J., \& d'Orsi, E. (2013). Fatores associados aos sintomas depressivos em idosos: estudo EpiFloripa. Revista de Saúde Pública, 47, 701-710.

Bretanha, A. F., Facchini, L. A., Nunes, B. P., Munhoz, T. N., Tomasi, E., \& Thumé, E. (2015). Sintomas depressivos em idosos residentes em áreas de abrangência das Unidades Básicas de Saúde da zona urbana de Bagé, RS. Revista Brasileira de Epidemiologia, 18, 1-12.

Gouveia, A. O., Dias, A. S., do Carmo Mercedes, B. P., da Costa Salvador, J., da Silva Junior, J. C. P., Peixoto, L. G., \& de Moraes, R. C. L. (2020). Detecção precoce dos sintomas depressivos pela equipe de saúde na atenção básica na região norte do país: revisão de literatura. Brazilian Journal of Development, 6(6), 38093-38103.

Souza, A. M. G., Santos Ferreira, T. L., Santos, K. M. R., Oliveira, D. J. D., \& Andrade, F. B. (2017). Avaliação da assistência à pessoa idosa na atenção primária à saúde: perspectiva de usuários. Revista Ciência Plural, 3(2), 42-52.

Didoné, L. S., Jesus, I. T. M. D., Santos-Orlandi, A. A., Pavarini, S. C. I., Orlandi, F. D. S., Costa-Guarisco, L. P., Gratão, A. C. M.; Gramany-Say, K., Cominetti, M. R., Gomes, G. A. O., \& Zazzetta, M. S. (2020). Fatores associados a sintomas depressivos em idosos inseridos em contexto de vulnerabilidade social. Revista Brasileira de Enfermagem, 73.

Faria, E. C., Silva, S. A. D., Farias, K. R. A. D., \& Cintra, A. (2011). Avaliação cognitiva de pessoas idosas cadastradas na estratégia saúde da família: município do Sul de Minas. Revista da Escola de Enfermagem da USP, 45(SPE2), 1748-1752.

Frade, J., Barbosa, P., Cardoso, S., \& Nunes, C. (2015). Depressão no idoso: sintomas em indivíduos institucionalizados e não-institucionalizados. Revista de Enfermagem Referência, (4), 41-49.

Gil, I. M. D. A., Costa, P. J. D. S., Cardoso, D. F. B., Parola, V. S. D. O., Almeida, M. D. L. F. D., \& Apóstolo, J. L. A. (2018). Eficácia da reminiscência na cognição, sintomas depressivos e qualidade de vida em idosos: protocolo de revisão sistemática. Revista de Enfermagem Referência, (16), 155-160.

Gullich, I., Duro, S. M. S., \& Cesar, J. A. (2016). Depressão entre idosos: um estudo de base populacional no Sul do Brasil. Revista Brasileira de Epidemiologia, 19, 691-701.

Hellwig, N., Munhoz, T. N., \& Tomasi, E. (2016). Sintomas depressivos em idosos: estudo transversal de base populacional. Ciência \& Saúde Coletiva, 21, $3575-3584$

Holz, A. W., Nunes, B. P., Thumé, E., Lange, C., \& Facchini, L. A. (2013). Prevalência de déficit cognitivo e fatores associados entre idosos de Bagé, Rio Grande do Sul, Brasil. Revista Brasileira de Epidemiologia, 16, 880-888.

Lentsck, M. H., Pilger, C., Schoereder, E. P., Prezotto, K. H., \& Mathias, T. A. D. F. (2015). Prevalência de sintomas depressivos e sinais de demência em idosos na comunidade. Revista Enfermagem, 17(3): 1-9.

Lima, T. J. V. D., Arcieri, R. M., Garbin, C. A. S., Moimaz, S. A. S., \& Saliba, O. (2014). Humanização na atenção básica de saúde na percepção de idosos. Saúde e Sociedade, 23, 265-276. 
Research, Society and Development, v. 10, n. 2, e23210212499, 2021

(CC BY 4.0) | ISSN 2525-3409 | DOI: http://dx.doi.org/10.33448/rsd-v10i2.12499

Lino, V. T. S. (2011). Triagem de problemas de saúde de idosos na atenção primária com um instrumento multidimensional (Tese de Doutorado).

Lino, V. T. S., Portela, M. C., Camacho, L. A. B., Rodrigues, N. C. P., Andrade, M. K. D. N., \& O'Dwyer, G. (2016). Rastreamento de problemas de idosos na atenção primária e proposta de roteiro de triagem com uma abordagem multidimensional. Cadernos de Saúde Pública, 32 , e00086715.

Magalhães, J. M., Carvalho, A. D. M. B., Carvalho, S. M., Alencar, D. D. C., Moreira, W. C., \& Parente, A. D. C. M. (2016). Depressão em idosos na estratégia saúde da família: uma contribuição para a atenção primária. REME Revista Mineira de Enfermagem, 1-6.

Martins, A. B., D'Avila, O. P., Hilgert, J. B., \& Hugo, F. N. (2014). Atenção Primária a Saúde voltada as necessidades dos idosos: da teoria à prática. Ciência \& Saúde Coletiva, 19, 3403-3416.

Matias, A. G. C., Fonsêca, M. D. A., Gomes, M. D. L. D. F., \& Matos, M. A. A. (2016). Indicadores de depressão em idosos e os diferentes métodos de rastreamento. Einstein (São Paulo), 14(1), 6-11.

Medeiros, K. K. A. S., Coura, A. S., \& Ferreira, R. T. (2017). O aumento do contingente populacional de idosos no Brasil e a atenção primária à saúde: uma revisão de literatura. Arquivos de Ciências da Saúde da UNIPAR, 21(3).

Molina, M. R. A. L., Wiener, C. D., Branco, J. C., Jansen, K., Souza, L. D. M. D., Tomasi, E., Silva, R. A., \& Pinheiro, R. T. (2012). Prevalência de depressão em usuários de unidades de atenção primária. Archives of Clinical Psychiatry (São Paulo), 39(6), 194-197.

Nogueira, E. L., Rubin, L. L., Giacobbo, S. D. S., Gomes, I., \& Cataldo Neto, A. (2014). Rastreamento de sintomas depressivos em idosos na Estratégia Saúde da Família, Porto Alegre. Revista de Saúde Pública, 48, 368-377.

Oliveira, M. F. D., Bezerra, V. P., Silva, A. O., Alves, M. D. S. C. F., Moreira, M. A. S. P., \& Caldas, C. P. (2012). Sintomatologia de depressão autorreferida por idosos que vivem em comunidade. Ciência \& Saúde Coletiva, 17, 2191-2198.

Pagliuca, L. M. F., Lima, B. D. S., Silva, J. M. D., Cavalcante, L. M., Martins, M. C., \& Araújo, T. L. D. (2017). Acesso de idosos às unidades de atenção primária à saúde. REME Revista Mineira de Enfermagem, 21, 1-5.

Pereira, A. S., Shitsuka, D. M., Parreira, F. J., \& Shitsuka, R. (2018). Metodologia da pesquisa científica. <http://repositorio.ufsm.br/bitstream/ha ndle/1/15824/Lic_Computacao_Metodologia-Pesquisa-Cientifica.pdf?sequence=1>.

Pereira, B. R. S., Lima, M. M. S., Salgueiro, C. D. B. L., Carvalho, V. P. S. (2019). Atuação da enfermagem frente à depressão na população idosa. Revista Enfermagem Digital Cuidado e Promoção da Saúde, 4(1), 51-56.

Piani, M. C., Alves, A. L. S., Bervian, J., Graeff, D. B., Pancotte, J., Doring, M., \& Dalmolin, B. M. (2016). Prevalência de sintomas depressivos em idosas de um Centro de Referência de Atenção ao Idoso no município de Passo Fundo, Rio Grande do Sul. Revista Brasileira de Geriatria e Gerontologia, 19(6), 930938.

Previato, G. F., Labegalini, C. M. G., Carreira, L., \& Baldissera, V. D. A. (2016). Características multidimensionais de saúde de idosos com sintomas depressivos. Revista Kairós: Gerontologia, 19(1), 339-357.

Ramos, G. C. F., Carneiro, J. A., Barbosa, A. T. F., Mendonça, J. M. G., \& Caldeira, A. P. (2015). Prevalência de sintomas depressivos e fatores associados em idosos no norte de Minas Gerais: um estudo de base populacional. Jornal Brasileiro de Psiquiatria, 64(2), 122-131.

Rêgo, A. D. S., Rissardo, L. K., Scolari, G. A. D. S., Sanches, R. D. C. N., Carreira, L., \& Radovanovic, C. A. T. (2017). Fatores associados ao atendimento a idosos por condições sensíveis à Atenção Primária à Saúde. Revista Brasileira de Geriatria e Gerontologia, 20(6), $773-784$.

Rodrigues, L. R., de Melo, A. T., dos Santos Ferreira, P. C., Dias, F. A., \& dos Santos Tavares, D. M. (2012). Qualidade de vida de idosos com indicativo de depressão: implicações para a enfermagem [Quality of life of the elderly with indication of depression: implications for nursing]. Revista Enfermagem UERJ, 20(6), 777-783.

Rodrigues, L. R., Tavares, D. M. D. S., Silveira, F. C. O., Dias, F. A., \& Martins, N. P. F. (2015). Qualidade de vida, indicativo de depressão e número de morbidades de idosos da zona rural. Revista de Enfermagem e Atenção à Saúde, 31-42.

Santos, M. C. S. D. (2013). Avaliação do déficit cognitivo e sua relação com características sociodemográficas, com condições de saúde e com o estilo de vida de pessoas idosas atendidas na Atenção Básica no município de Jacareí, São Paulo (Dissertação de Mestrado).

Silva, A. R., Sgnaolin, V., Nogueira, E. L., Loureiro, F., Engroff, P., \& Gomes, I. (2017). Doenças crônicas não transmissíveis e fatores sociodemográficos associados a sintomas de depressão em idosos. Jornal Brasileiro de Psiquiatria, 66(1), 45-51.

Silva, G. É. M. D., Pereira, S. M., Guimarães, F. J., Perrelli, J. G. A., \& Santos, Z. C. D. (2014). Depressão: conhecimento de idosos atendidos em unidades de saúde da família no muncípio de Limoeiro-PE. Revista Mineira de Enfermagem, 18(1), 82-93.

Soares, S. M., Silva, P. A. B., Santos, J. F. G., \& Silva, L. B. (2017). Associação entre depressão e qualidade de vida em idosos: atenção primária à saúde [Association between depression and quality of life in older adults: primary health care]. Revista Enfermagem UERJ, $25,19987$.

Uchoa, V. S., Chaves, L. L., Botelho, E. P., Polaro, S. H. I., \& Oliveira, M. D. F. V. (2019). Fatores associados a sintomas depressivos e capacidade funcional em idosos. Cogitare Enfermagem, 24. 